\title{
Le poisson dans l'alimentation du vietnamien
}

\author{
$2^{\mathrm{e}}$ partie

\section{Conserves appertisées, farines et saucisses de poisson}

\author{
par C. RICHARD, NGUYEN NHU NGHI, NGUYEN THI LAU \& F. LITALIEN
}

Dans un travail antérieur publié dans cette revue (8), nous avions étudié la composition chimiqueet les propriétés nutritives des 19 espèces de poisson les plus couramment consommées au Viet-Nam, et attiré l'attention sur la valeur alimentaire des 3 principales productions ichtyologiques vietnamiennes traditionnelles: nuoc mam, mam ca et ca kho. Rappelons que les nuoc mam, solutions hypersalées d'amino-acides résultent du protéolysat de la chair de poissons entiers, que les mam ca, présentés sous enrobage de sucre ef de riz grillé, sont obtenus par macération de poissons éviscérés et étêtés dans du sel et que les ca kho correspondentà des poissons salés et séchés.

La présente étude intéresse plus particulièrement des produits de transformation et de conservation du poisson, fabriqués par le Service tect.nique des industries des pêches du gouvernement vietnamien (par abrévation S. T. I. P. G. V. N.). II ne s'agit pas ici de préparations artisanales comme le sont les nuoc mam, mam ca et ca kho, mais de véritables produits industriels qui nécessitent l'emploi d'un équipement électroméccnique spécial.

Nous nous sommes limités dans cette note à l'examen de 3 types de produits :

10 Conserves appertisées de poisson (Ophiocephalus striatus et Clupea (Harengula) perforatc) préparées selon le goût vietnamien, c'est-à-dire additionnées de nuoc mom, de nlioc tuong (sauce de soja) et d'aromates divers.

$2^{\circ}$ Forines de poisson (Stolephorus commersoni

Reçu pour publication : novembre 1960.

Rev. Elev. Méd. vét. Pays trop., 1960, 13, no 4. ef Sourida tumbil) présentées dans des sachets en matière plastique.

30 Saucisses de poisson (Notopterus notopterus ef Chirocentrus dorab) également conditionnées sous emballage en matière plastique.

Les essais entrepris par le Service technique des pêches méritent d'être encouragés, car les produits de transformation du poisson fabriqués par cet organisme permettent de résoudre le difficile problème de la conservation et du transport du poisson, denrée hautement altérable et périssable surtout en région tropicale où il n'existe généralement pas de chaînes de froid. On peut ainsi garder intacte sous un volume réduit la valeur nutritive des poissons à des disances très éloignées des centres de pêche, ef árnir à peu de frais et sous forme concentrée une source intéressante de protéines animales à des populations dont les rations principalement composées d'aliments de nature glucidique sont trop souvent carencées en protéines, principalement en protéines d'origine animale nécessaire aux besoins énergétiques ef plastiques de l'organisme.

Il nous a donc paru intéressant de rapporter dans ce travail le mode de fabrication, la composition chi mique, la qualité bactériologique, la valeur alimentaire et énergétique de chacun des trois types de produits préparés industriellement par le S. T. I. P. G. V. N. En outre les tests d'acceptabilité, les utilisations culinaires possibles et les débouchés commerciaux qu'ils peuvent offrir ont été étudiés dans chaque cas particulier.

Sur le plan analytique, nous avons utilisé les mêmes techniques que celles précédemment décrites (8). 
TABLEAU I

Conserves appertisées de poisson, préparées selon le goût vietnamien.

Caractères organoleptiques, bactérlologiques et chimiques - Valeur énergétique.

\begin{tabular}{|c|c|c|c|c|c|c|}
\hline Echantillons & $\begin{array}{l}\text { CA } \\
\text { Conserve de CA IOC } \\
\text { Opiocephalus stria- } \\
\text { tus au Nuoc Mam et } \\
\text { au Nuoc Tuong }\end{array}$ & $\begin{array}{c}\text { CB } \\
\text { Conserve de CA TRICH } \\
\text { Clupea (Harengula) } \\
\text { Derforata au Nuoc } \\
\text { Mam et au Nuoc Tuone }\end{array}$ & \begin{tabular}{l}
\multicolumn{1}{c|}{ CC } \\
Conserve de CA THICH \\
Clupea (Harengula) \\
Derforata au Nuoc \\
Tuong
\end{tabular} & $\begin{array}{l}\text { CD } \\
\text { Conserve de CA TRJCH } \\
\text { Clupea (Harengula) } \\
\text { perforata au Nuoc } \\
\text { Mam et aux aromates } \\
\text { vietnamiens }\end{array}$ & $\begin{array}{l}\qquad \mathrm{CE} \\
\text { Conserve de CA TRTCH } \\
\text { Clupea (Harengula) } \\
\text { perforata au Nuoc } \\
\text { Mam et aux aromates } \\
\text { vietnamiens }\end{array}$ & $\begin{array}{l}\text { CF } \\
\text { Conserve de CA TRICH } \\
\text { Clupea (Barengula) } \\
\text { perforata au Nuoc } \\
\text { Mem et aux arowates } \\
\text { vietnamiens }\end{array}$ \\
\hline$\frac{\text { Caractères organoleptiques }}{\text { du produit conservé }}$ & Satisfaisants & Satisfaisants & Satisfaisants & Satisfaisants & Satisfaisants & Satisfaisants \\
\hline Etat de l'étamage intérieur & Attaqué & Légèrement attaqué & $\begin{array}{l}\text { Très légèrement } \\
\text { attaqué }\end{array}$ & In assez bon état & En assez bon état & En assez bon état \\
\hline 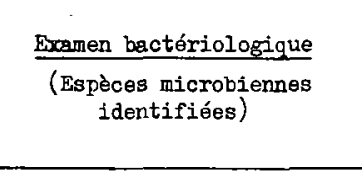 & $\begin{array}{l}\text { Absence de gernes } \\
\text { (Produit stérile) } \\
\text { Très bonne qualité } \\
\text { bactériologique }\end{array}$ & $\begin{array}{c}\text { Saroines } \\
\text { Bonne qualitité } \\
\text { baotériologịue }\end{array}$ & $\begin{array}{l}\text { Baaillus } \\
\text { Bonne qualité } \\
\text { bactériologique }\end{array}$ & $\begin{array}{c}\text { Sarcines } \\
\text { Bonne qualité } \\
\text { bactériologique }\end{array}$ & $\begin{array}{l}\text { Sarcines } \\
\text { Anaérobies sulfito- } \\
\text { réducteurs. } \\
\text { Qualité. bactériolo- } \\
\text { gi que douteuse. }\end{array}$ & $\begin{array}{l}\text { Sarcines } \\
\text { Anaérobies sulfito- } \\
\text { réducteurs. } \\
\text { Qualité bactériolo- } \\
\text { Eique douteuse }\end{array}$ \\
\hline 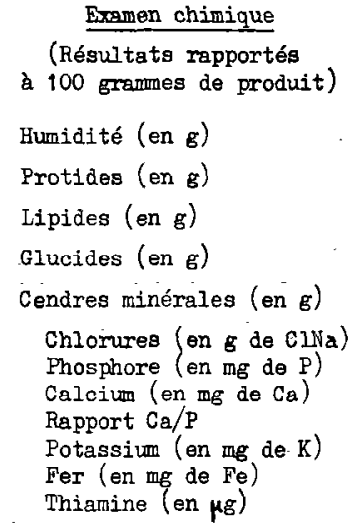 & $\begin{array}{c}51,96 \\
18,62 \\
23,16 \\
2,51 \\
3,75 \\
2,22 \\
220 \\
280 \\
1,27 \\
376 \\
0,17 \\
25\end{array}$ & $\begin{array}{c}60,01 \\
19,95 \\
7,77 \\
5,76 \\
6,51 \\
4,33 \\
350 \\
469 \\
1,34 \\
255 \\
0,17 \\
40^{-}\end{array}$ & $\begin{array}{c}59,58 \\
20,12 \\
-\quad 8,29 \\
=\quad 5,72 \\
6,29 \\
4,09 \\
395 \\
492 \\
1,24 \\
235 \\
0,20 \\
35\end{array}$ & $\begin{array}{c}66,36 \\
20,56 \\
1,31 \\
5,47 \\
6,30 \\
4,09 \\
400 \\
501 \\
1,25 \\
209 \\
0,22 \\
25\end{array}$ & $\begin{array}{c}64,96 \\
22,05 \\
2,77 \\
4,38 \\
5,84 \\
3,57 \\
380 \\
463 \\
1,21 \\
251 \\
0,21 \\
15\end{array}$ & $\begin{array}{c}69,70 \\
19,81 \\
2,44 \\
1,96 \\
6,09 \\
3,80 \\
385 \\
460 \\
1,19 \\
273 \\
0,20 \\
25\end{array}$ \\
\hline $\begin{array}{l}\text { Valeur énergétique } \\
\text { (en calories et rap- } \\
\text { portée à } 100 \\
\text { g })\end{array}$ & 293 & -173 & 178 & 116 & 131 & 109 \\
\hline $\begin{array}{c}\text { Poids net } \\
\text { du produit conservé } \\
\text { Calories par boite }\end{array}$ & $\begin{array}{l}324 \\
949 .\end{array}$ & $\begin{array}{l}132 \\
228\end{array}$ & $\begin{array}{l}124 \\
221\end{array}$ & $\begin{array}{l}196 \\
134\end{array}$ & $\begin{array}{l}110 \\
144\end{array}$ & $\begin{array}{l}117 \\
128\end{array}$ \\
\hline
\end{tabular}




\section{I. - CONSERVES APPERTISÉES}

Alors que les conserves de poisson, originaires d'Europe ou du Maroc, telles que les sardines à l'huile ou à la tomate, n'ont trouvé en ExtrêmeOrient qu'un débouché assez restreint, les conserves appertisées d'Ophiocephalus striatus (échantillon $C A$ ) ou de Clupea (Harengula) perforata (échantillons $C B$ à $C F$ ) présentées en boîte métallique soudée par le S. T. I. P. G. V. N. trouveront, a notre avis, rapidement leur place sur la table des Vietnamiens, dès qu'elles seront préparées en quantité suffisante. Elles ont été tout spécialement étudiées et composées pour pouvoir être consommées avec le riz et les diverses préparations végétariennes courantes. En effet. accompagnées des 2 sauces condimentaires traditionnelles nuoc mam et nuoc fuong, ef d'aromates de la flore locale, elles conviennent parfaitement au goût et aux habitudes culinaires des Vietnamiens et probablement d'un grand nombre d'Extrême-Orientaux. Nous pensons même qu'elles pourront être exportées avec succès sur le Cambodge et le Laos.

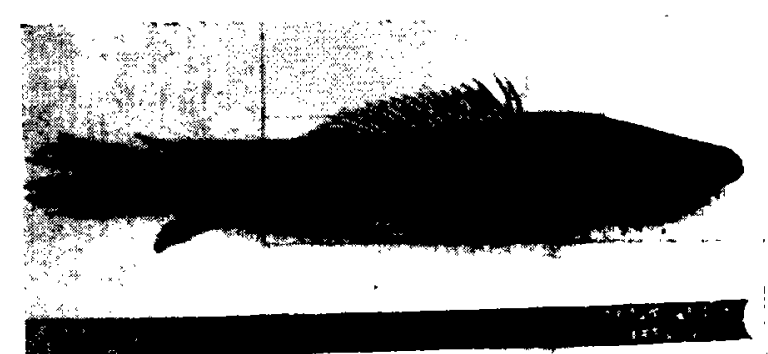

Fig. 1. - Ophiocepholus striotus ( $\mathrm{Ca}$ lor).

\section{a) Composition ef mode de fabrication}

Nous avons examiné 6 échantillons de conserves appertisées de poisson, de type vietnamien, répertoriées de CA à CF (Tableau I).

L'échantillon CA a été préparé à partir d'un poisson d'eau douce Ophiocephalus striotus (fig. $n^{\circ} 1$ ), de la famille des Ophiocephalidae (nom vernaculaire vietnamien $\mathrm{Ca}$ Loc) coupé menu frit à I'huile d'arachide en même temps que de petits morceaux de porc (en vietnamien Thit Heo) et additionné de sauce nuoc mam, autolysat salé de chair de poisson (2-11) et de nuoc fuong, sauce de soja obtenue après 3 mois d'hydrolyse enzymatique ( $(7)$.
Les échantillons CB d CF correspondent à des conserves de sardines vietnamiennes Clupeo (Harengulo) perforoto, Clupeidoe (nom vernaculaire vietnamien $(a$ Trich), étêtées et éviscérées, auxquelles il a été ajouté du nuoc mam et du nuoc tuong (échantillon CB), du nuoc tuong (échantillon (C), du nuoc mam et divers aromates: clous de girofle, poudre de cannelle et de poivre, ef du sucre (échantillons CD, CE et CF).

Toutes ces conserves en boîte métallique soudée ont été stérilisées par autoclavage à $115^{\circ} \mathrm{C}$ pendant 30 minutes, à l'exception des échantillons CF et CE qui furent traités seulement à $100^{\circ}$ pendant 15 minutes.

\section{b) Valeur alimentaire et nutritive}

Nous avons groupé dans le tableau I les résultats ces examens chimiques, résultats rapportés à 100 grammes de produit, homogénéisé pour les besoins de l'analyse.

Outre les 3 grands groupes de principes alimentaires: protides, lipides, glucides, nous cvons dosé les cendres minérales, les chlorures, les ions Phosphore, Calcium, Fer et Potassium, ainsi que la vitamine $B_{1}$. Les valeurs énergétiques de ces conserves, exprimées en calories et rapportées à 100 grammes de produit d'une part, et au contenu des boîtes d'autre part, ont été calculées à l'aide des coefficients de transformation calorifique d'Atwater.

Enfin dans le tableau $I I$, nous avons indiqué, à tiire comparatif, les caractéristiques chimiques et la valeur énergétique de 2 échantillons de scrdines marocaines à l'huile et à la tomate, analysés dans notre laboratoire (échantillons $M_{1}$ et $M_{2}$ ).

Ces conserves de poisson à la vietnamienne sont caractérisées par une teneur élevée en matières protéiques (18.5 à 22 p. 100), légèrement supérieure à celle des sardines marocaines à I'huile ef à la tomate que nous avons examinées (15 à 19 p. 100). Elles sont conformes aux exigences de l'inspection technique des subsistances de l'armée française qui recommande pour les conserves de sardine à l'huile la composition centésimale suivante (3) :
protides ......... 18,8 p. 100
lipides ......... 32,2 p. 100
calcium (Ca)..... 0, 0,5 p. 100
phosphore (P).... 0, 0,4 p. 100 
TABLEAU II

Conserves de sardines marooaines à l'huile at à la sauce tomate

\begin{tabular}{|c|c|c|}
\hline Echantillons & M $i$ & M 2 \\
\hline$\frac{\text { Caractères organoleptiques }}{\text { du produit conservé }}$ & Satisfaisants & Satisfaisants \\
\hline Etat de l'étamage intérieur & En assez bon état & Légèrement attaqué \\
\hline $\begin{array}{l}\text { Examen chimique } \\
\text { (Résultats repportés } \\
\text { à } 100 \text { grammes de produit) } \\
\text { Humidité (en g) } \\
\text { Frotides (en } \mathrm{g} \text { ) } \\
\text { Lipides (en g) } \\
\text { Glucides (en g) } \\
\text { Cendres minérales (en g) } \\
\text { Chlorures (en g de ClNa) } \\
\text { Phosphore (en mg de P) } \\
\text { Calcium (en mg de Ca) } \\
\text { Rapport Ca/P } \\
\text { Potassium (en mg do } \mathrm{K} \text { ) }\end{array}$ & $\begin{array}{c}69,78 \\
15,05 \\
8,85 \\
4,07 \\
2,25 \\
1,81 \\
139 \\
- \\
- \\
298\end{array}$ & $\begin{array}{c}59,30 \\
19,25 \\
15,74 \\
2,98 \\
2,73 \\
1,23 \\
353 \\
500 \\
1,41 \\
365 .\end{array}$ \\
\hline $\begin{array}{c}\text { Valeur énergétique } \\
\text { (en calories ot: rapportée } \\
\text { à } 100 \text { grammes) }\end{array}$ & 156 & 231 \\
\hline $\begin{array}{l}\text { Poids net du produit conservé } \\
\text { Calories par boîte }\end{array}$ & $\begin{array}{l}125 \mathrm{~g} \\
195 \mathrm{cal}\end{array}$ & $\begin{array}{l}126 \mathrm{~g} \\
\quad 291 \mathrm{cal}\end{array}$ \\
\hline
\end{tabular}

fer $(\mathrm{Fe}) \ldots \ldots \ldots$

Vitamine $B_{1} \ldots \ldots$

1,3 milligramme

50 microgrammes

Valeur ćnergétique. 344 calories.

Comme il fallait s'y attendre, le pourcentage en protides des échantillons CA à CF (Tableau i) est du même ordre de grandeur que celui des poissons vietnamiens de consommation courante (12 à 25 p. 100) et nettement inférieur à celui des farines de poisson destinées à l'alimentation humaine que nous étudierons plus loin (50 à 70 p. 100). Comme les besoins quotidiens en protides du Vietnamien dont le poids corporel moyen n'est que de 55 kilos sont largement couverts par 70 grammes de protides dont 30 grammes d'origine animale, ces conserves de poisson par leur haute teneur en matières albuminoïdes peuvent concourir efficacement à assurer les exigences d'une alimentation rationnelle. De plus, il n'est pas inutile de rappeler que les protides de poisson contiennent, en proportions adéquates, les amino-acides indispensables, et $\mathrm{cn}$ particulier la lysine, qui fait défaut aux régimes à base de céréale.

Quant aux lipides, si j'on excepte l'échantillon CA préparé à l'huile d'arachide et additionné de petits morceaux de porc thit heo, leur teneur est comprise entre 2 ef 6 p. 100 (Echantillons CB à $C F$ ) alors qu'elle varie généralement entre 15 et 30 p. 100 dans la plupart des conserves de sardines par exemple. 11 en résulte finalement pour ces conserves vietnamiennes une valeur énergétique moindre, mais en climat tropical où l'homme n'a pas besoin de lutter contre le froid, l'apport de lipides peut être considérablement réduit par rapport aux normes européennes et américaines. A notre avis, ce pourcentage en matières grasses relativement faible serait plutôt 
un avantage qu'un inconvénient, compte tenu des considérations locales.

Par contre, les conserves de poisson du S. T.I.P. G. V.N., et plus spécialement les échantillons CB a CF, renferment plus de sels minéroux que les sardines marocaines que nous avons examinées (Tableau II) ; ceci provient surtout du chlorure de sodium et des quelques autres ionsminéraux apportés par les sauces condimentaires salées nuoc mam et nuoc tuong qui entrent dans leur composition (Tableaux I ef II) : 6 p.100 de sels minéraux et 4 p. 100 de $\mathrm{CINa}$, contre 2,5 p. 100 de sels minéraux et 1,5 p. 100 de CINa dans les sardines marocaines. Cet apport minéral permet de lutter contre la déperdition saline par sudation, assez importante sous les Tropiques.

A cela, il faut ajouter l'importance de l'apport phosphocalcique qui atteint presque 0,5 p. $100 \mathrm{de}$ calcium et 0,4 p. 100 de phosphore, et surtout la valeur du rapport $C A / P$, rapport qui pour les 6 échantillons $C A$ à $C F$ a une valeur moyenne de 1,25. Pour mémoire, rappelons que la chair des mammifères ( $\mathrm{Ca} / \mathrm{P}$ compris entre 0,05 et 0,10 ) ef les céréales alimentaires $(\mathrm{Ca} / \mathrm{P}=0,2)$ sont caractérisées par un déséquilibre de ce rapport par insuffisance de calcium. Les nutritionistes de nombreux pays recommandent en effet, pour que le calcium et le phosphore alimentaires s'équilibrent convenablement, les valeurs suivantes pour ce rapport :

$\mathrm{Ca} / \mathrm{P}=0,8$ (régime des adultes)

$\mathrm{Ca} / \mathrm{P}=1$ à 1,2 (régime des enfants, des femmes enceintes ef allaitantes).

En ce qui concerne la vitamine $B_{1}$ (thiamine) nous n'avons pas été surpris de trouver des chiffres aussi bas que ceux obtenus lors de l'étude des 19 espèces de poissons vietnamiens de consommation courante -20 à 50 microgrammes pour 100 grammes - taux nettement plus faibles que ceux des viandes rouges deboucherie - en moyenne 200 microgrammes pour 100 grammes. Il est intéressant de noter que les échantillons $C B$ et $C C$ contenant de la sauce de soja nuoc tuong en renferment davantage que les 4 autres échantillons (CC:45, CB: $40, C A$ : 25. $C D: 25, C F: 25$ et $C E: 15$ microgrammes pour 100 grammes). Il n'est pas inutile de souligner l'intérêt des sauces de soja, relativement riches en thiamine, dans les rations hyperglu- cidiques des Extrême-Orientaux. Du reste, il y a quelques années nous eûmes à analyser le contenu d'une boîte de ration militaire préparée par le service des subsistances japonais, ration à base de riz blanc, de sauce de soja et de poisson genre thon, dont nous rapportons ci-dessous l'analyse :

\begin{tabular}{|c|c|c|}
\hline & 56,79 & . 10 \\
\hline ofides & 11,78 & 》 \\
\hline & 0,10 & \\
\hline trat & 29,14 & 1) \\
\hline héraux & 2,19 & \\
\hline $\mathrm{Na}$ & 1,42 & \\
\hline
\end{tabular}

Thiamine 100 microgrammes $p .100 \mathrm{~g}$ Valeur énergétique : 164,58 calories p. $100 \mathrm{~g}$.

En tenant compte du poids net de cette boîte (390 g) la quantité totale de thiamine apportée par cette conserve correspondait à 390 microgrammes, couvrant ainsi le quart des besoins en vitamine $B_{1}$ de la ration de l'Asiatique que l'on peut chiffrer par jour à 1.500 microgrammes au minimum.

\section{c) Qualité bactériologique et limite du temps de conservation}

Les échantillons CE et CF à la suite d'une stérilisation insuffisante renferment des germes anaérobies sulfito-réducteurs et leur qualité bactériologique peut être considérée comme douteuse, par contre les autres échantillons CA à CD correctement autoclavés, exempts de germes dangereux ou pathogènes, sont de bonne qualité bactériologique.

En ce qui concerne les limites du temps de stockage, nous croyons sage de les fixer à 1 an, car comme nous avons pu déjà le constater, le nuoc mam et les sauces de soja attaquent à la longue les éfamages intérieurs ce qui déprécie les caractères organoleptiques des produits conservés. Toutefois, il faut signaler que les étamages vernissés résistent beaucoup mieux à l'action de ces 2 sauces condimentaires.

\section{II. - FARINES DE POISSON DESTINÉES A L'ALIMENTATION HUMAINE}

Comme nous l'avons déjà indiqué (8), il entre dans la composition de la chair des poissons de 70 à 85 p. 100 d'eau ef de 0,1 à 13 p. 100 de matières grasses. On comprend donc tout l'in- 
térêt que présentent pour l'alimentation les forines de poisson obtenues après élimination de l'eau et des lipides par des moyens quelquefois artisanaux (5), le plus souvent industriels. II s'en suit'que ces farines de poisson constituent des aliments à haute teneur en protéines animales. Suivant l'origine et les parties de poisson utilisées, elles renferment aussi plus ou moins d'élément's minéraux, en particulier des ions calcium et phosphore.

Si les farines de poisson destinées à l'alimentation des animaux - porcs, bovins, ovins, volaille - dans le but de supplémenter leurs rations en amino-acides indispensables (lysine) sont fabriquées et utilisées avec profit depuis presque un siècle (1), it n'y a guère plus d'une dizaine d'années que l'on a proposé pour la consommation humaine des poudres de poisson frais déshydratées, désodorisées ou non (9). Elles ont été introduites dans le régime de populations sous-développées, carencées en protéines animales, sous forme d'aliments composés : farine de mil-arachide-poisson au Sénégal (10) ; «protein flour process » de Vries au Congo, la dessiccation étant effectuée en présence d'une substance amylacée fixant l'humidité par adsorption (6); pain ou sauces condimentaires auxquels on incorpore de la farine de poisson (9).

Les farines à usage vétérinaire dont la production mondialc dépasse 400.000 tonnes par an (1) sont préparées soit à partir de poissons entiers après extraction de l'huile, soit à partir de déchets de poisson, soit encore à partir de poissons non comestibles pour l'homme.

Celles destinées à la consommation humaine sont fabriquées avec des poissons comestibles, maigres de préférence, entiers ou simplement éviscérés, de façon à minimiser les pertes en éléments nutritifs et à obtenir un bon apport phospho-calcique.

II va de soi que l'efficacité alimentaire de ces farines, dans l'un et l'autre cas, dépend de l'origine et de la qualité des tissus de poisson utilisés ef également de la nature des traitements industriels subis (1).

Des nombreux travaux auxquels ont donné lieu les farines de poisson employées dans la supplémentation des rations animales, on peut retenir :

10 Théoriquement, seuls les poissons ou déchets frais devraient être traités et il serait souhaitable que les, matières altérées soient. réservées à la fabrication d'engrais pour l'agriculture. II faut donc éviter un stockage prolongé des poissons avant leur transformation en farines, alimentaires.

$2^{\circ}$ Au cours du traitement industriel, après la cuisson du poisson et le passage à la presse qui élimine la plus grande partie des lipides et de l'eau, il faut apporter un soin tout particulier à la déshydratation finale, car un chauffage trop poussé entraîne une diminution du taux de lysine. Toutes choses étant par ailleurs égales, un séchage à basse température sous vide partiel permet d'obtenir de meilleures farines qu'un séchage à température moyenne $\left(60^{\circ}-105^{\circ} \mathrm{C}\right)$ par l'emploi de la vapeur ou de l'eau chaude circulant dans une double paroi, ce dernier procédé étant luimême préférable à une dessiccation dà haute température $\left(200^{\circ}-320^{\circ} \mathrm{C}\right)$ obtenue par contact direct de la flamme ou de l'air surchauffé (4).

3o Compte tenu des diverses réglementations nationales relatives à ces farines, on pourra se baser sur les normes suivantes pour déterminer la qualité et conclure à l'efficacité de ces produits:

- matières protéiques : plus de 45 p. 100 (62 à 80 p. 100 pour les meilleures qualités).

- matières grasses : moins de 10 p. 100 (moins de 5 p. 100 pour les meilleures qualités).

- humidité : moins de 10 p. 100 (si possible de 5 p. 100).

L'observation de ces normes permet de limiter grandement les risques d'altération pendant le stockage. De toute façon, le stockage ne devrait pas dépasser 1 à 2 mois. Si l'on doit craindre un rancissement possible, au cas ou les taux des lipides ne serait pas négligeable, les délais de conservation devraient être encore réduits. On veillera également à ce que le conditionnement soit effectué en boîte ou en sachets hermétiques que l'on entreposera dans un lieu aussi sec que possible, car il ne faut pas oublier que les poudres de poisson sont très hygroscopiques et que lorsque leur humidité dépasse 10 p. 100 , elles favorisent le développement de microorganismes, cause d'altérations diverses.

Des farines de poisson destinées à la consommation humaine ont été préparées tout récemment au Viet-Nam de façon industrielle par le S. T. I. P. G. V.N. Elles se présentent sous 2 aspects : 
TABIEAUU III

Farines de poisson destinées à l'alimentation humaine.

Caractères bactériologiques et chimiques. Valeur énergétique.

\begin{tabular}{|c|c|c|c|c|c|c|c|}
\hline Echantillons & $\begin{array}{l}\text { Poudre de polsaon } \\
\text { Ndakele } \\
\text { fabriquée par } \\
\text { 1'OVIPARU } \\
\text { (Ruanda-Urunds) }\end{array}$ & $\begin{array}{l}\text { H. A. } \\
\text { Youdre de CA COM } \\
\text { Stolophorus oom- } \\
\text { mersond addition. } \\
\text { née d'aroms tes } \\
\text { et do sauce de } \\
\text { soja (Xi-Dau) }\end{array}$ & $\begin{array}{c}\text { P. B. } \\
\text { Poudre de CA COM } \\
\text { Stolephorus oom- } \\
\text { mergoni addition- } \\
\text { née de Nuoc Mam }\end{array}$ & $\begin{array}{l}\text { P. C. } \\
\text { Poudre de CA COM } \\
\text { Stolephorus oom- } \\
\text { mersont }\end{array}$ & $\begin{array}{c}\text { G. D. } \\
\text { Granulés de CA MOI } \\
\text { Saurida tumbj] } \\
\text { additionnés do } \\
\text { oondimente et } \\
\text { d'aromates divero }\end{array}$ & $\begin{array}{c}\text { G. F. } \\
\text { Cranulós do CA NOI } \\
\text { Sauride tumbil } \\
\text { additionnés de } \\
\text { condiments et } \\
\text { dtaromites divers }\end{array}$ & $\begin{array}{c}\text { G. F. } \\
\text { Granulés de CA MOI } \\
\text { Saurida tumbil } \\
\text { additionnós de } \\
\text { condiments ot } \\
\text { d'aromates divers }\end{array}$ \\
\hline $\begin{array}{l}\text { bxamen hnctériologdque } \\
\text { (Espèces microbiennes } \\
\text { identifíes) }\end{array}$ & & Baoillus & Baoillus & $\begin{array}{l}\text { Baofllus } \\
\text { Aerobacter } \\
\text { aerogenes }\end{array}$ & Becillus & Bacillus & $\begin{array}{c}\text { Barlilus } \\
\text { flavobacterium }\end{array}$ \\
\hline 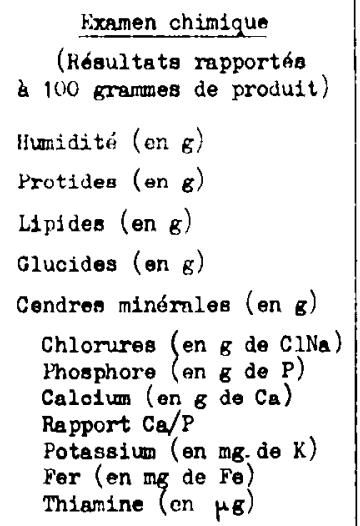 & $\begin{array}{c}8,40 \\
68,10 \\
11,00 \\
0 \\
12,5\end{array}$ & $\begin{array}{r}6,72 \\
61,25 \\
3,65 \\
6,78 \\
21,60 \\
7,31 \\
2,40 \\
3,99 \\
1,66 \\
340,70 \\
9,24 \\
45\end{array}$ & $\begin{array}{r}9,52 \\
62,19 \\
3,64 \\
5,01 \\
19,64 \\
5,41 \\
2,50 \\
4,22 \\
1,68 \\
205,60 \\
19,60 \\
40\end{array}$ & $\begin{array}{c}11,10 \\
69,811 \\
4,60 \\
0 \\
14,49 \\
1,71 \\
2,56 \\
5,09 \\
1,98 \\
106,20 \\
20,16 \\
30\end{array}$ & $\begin{array}{c}4,96 \\
38,00 \\
9,13 \\
7,97 \\
19,92 \\
7,17 \\
2,80 \\
3,45 \\
1,23 \\
584 \\
11,06 \\
50\end{array}$ & $\begin{array}{c}6,06 \\
54,75 \\
12,99 \\
8,76 \\
18,65 \\
6,73 \\
1,92 \\
3,18 \\
1,65 \\
564 \\
10,92 \\
50\end{array}$ & $\begin{array}{r}8,21 \\
48,75 \\
14,70 \\
10,29 \\
18,05 \\
6,87 \\
1,80 \\
2,79 \\
1,50 \\
624 \\
8,68 \\
60\end{array}$ \\
\hline 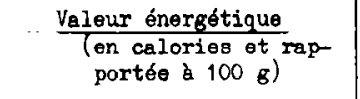 & $371, \overline{4}$ & 304,97 & 301,56 & 320,64 & 346,05 & 366,11 & 368,46 \\
\hline
\end{tabular}


- des poudres impalpables de couleur brun clair, à odeur de poisson (Tableau III, Echantillons PA, PB, PC);

- des granulés, d'un diamètre de 1 à $2 \mathrm{~mm}$, présentant sur fond brun des colorations ponctiformes jaunes, vertes ef rouges (Tableau III; Echantillons GD, GE, GF).

A la différence des produits similaires préparés au Congo ex-Belge (6-9) la composition de ces farines, comme celle des conserves de poisson examinées plus haut, a été étudiée pour satisfaire les goûts et habitudes alimentaires des Vietnamiens. Pour cette raison, elles ont été additionnées de nuoc mam, de sauce de soja, de divers aromates ef condiments.

\section{a) Composition ef mode de fabrication}

Les 3 échantillons de poudre que nous avons examinés, (Tableau III, échantillons PA. PB. PC) ont été préparés à partir de Stolephorus commersoni (fig. no 2), Clupeidae (nom vernaculaire

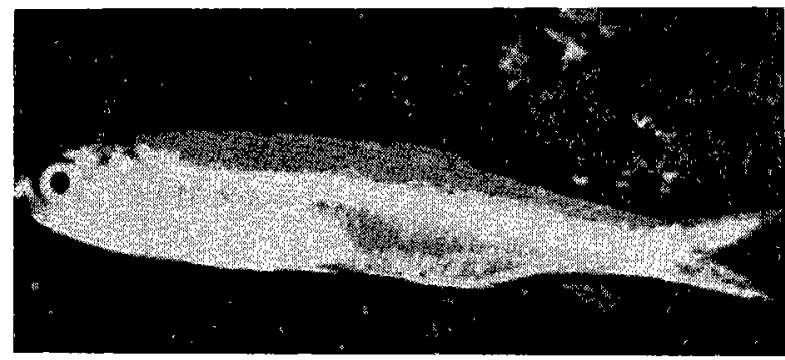

Fig. 2. -- Stolephorus commersoni (Ca com).

vietnamien (a Com) additionné de sauce de soja concentrée $X i$-Dau, d'aromates divers: clous de girofle, poudre de cannelle ef de poivre, et de sucre (échantillon PA), ou simplement aromatisé au nuoc mam (échantillon $P B$ ) ou encore tel quel (échantillon PC).

Les 3 échantillons de granulés de poisson étudiés (Tableau III, échantillons GD, GE, GF) sont à base de Saurida tumbil (fig. no 3), Scopelidae (nom vernaculaire vietnamien $\mathrm{Ca} m o i$ ), d'un assez grand nombre de condiments, et diversement colorés.

L'arôme et la saveur spéciale de ces condiments sont obtenus par addition de :

- granulés GD : piment en poudre, poivre, sel, cinq épices, glutamate de sodium, sucre, nuoc mam, sauce de soja concentrée, XiDou, agar en poudre, sésame, feuilles aromatiques tia tô.

- granulés GE : piment en poudre, poivre, cinq épices, glutamate de sodium, sucre, sauce de soja, sel, poudre d'agar, réglisse, extrait de rành rành (colorant végétal jaune).

- granulés GF : piment en poudre, cinq épices, poivre, glutamate de sodium, sucre, sésame, citronnelle, sauce de soja, sel, réglisse, poudre d'agar, colorant végétal rouge, extrait de rònh rành.

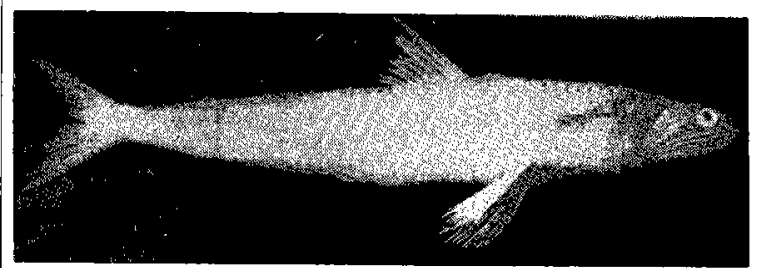

Fig. 3. - Saurita tumbil (Ca moi).

Quant à la fabrication industrielle de ces farines de poisson destinées à la consommation humaine, elle a pu être réalisée grâce qu matériel d'équipement fourni au S. T. I. P. G.V.N. par l'Aide américaine au Viet-Nam (USOM). L'installation électromécanique comprend 2 machines essentielles: une presse, press for fish cake ef un moulin-broyeur, grinding machine. Le poisson, cuit rapidement dans l'eau pour le débarrasser des matières grasses, est essoré et comprimé au moyen de la presse, puis passé au séchoir. Il est ensuite broyé dans la grinding mochine et transformé en farine plus ou moins fine.

Le S.T.I.P.G. V. N. dispose en outre d'un atomiseur qui transforme en poudre toute solution ou tout liquide alimentaire (nuoc mam, sauce de soja ...) et d'un groupe frigorifique - modèle de laboratoire - quï permet la fabrication de filets de poissons congelés.

\section{b) Valeur alimentaire ef nutritive}

La composition chimique ef la valeur énergétique de ces farines: poudres $\mathrm{PA}, \mathrm{PB}, \mathrm{PC}$ et granulés GD, GE, GF sont rapportées dans le Tableau III.

Ces denrées sont caractérisées avant tout par leur houte teneur en protéines et en sels minéraux, particulièrement en calcium et en phosphore. 
Le pourcentage en matières protéiques des poudres PA, PB, PC compris entre 61 et 70 p. 100 correspond, selon les normes indiquées plus haut, à des farines de poisson de bonne qualité. La farine de Stolephorus (PC) est comparable. au point de vue de la valeur en protéines, à celle de poisson Ndakalc préparée au Ruanda-Urundi par I'OVIPARU (Tableau III) (9). On peut remarquer au passage que ces poudres renferment environ 3 fois 1.2 plus de protéines que les poissons à partir desquels elles sont fabriquées.

Quant aux granulés GD, GE, GF, par suite de la forte proportion d'ingrédients, aromates et condiments qui entrent dans leur composition, leur taux de protéines est moindre : 48-58 p. 100. tout en étant encore conformes aux normes de référence.

L'apport de matières minérales (14,5 à 21,5 p. p. 100) doit être signalé aussi, et plus spécialement la très forte proportion de calcium $(2.7$ à 5 p. 100) et de phosphore (1,8 d̀ 2,8 p. 100), ainsi que le rapport $\mathrm{Ca} / \mathrm{P}$ : valeur moyenne 1,60 . On voit donc que l'adjonction de ces farines à des rations à base de céréales pour lesquelles ce ropport a une valeur inverse, permet de compenser le déséquilibre des ions $C a$ et $P$.

On peut remarquer aussi la teneur relativement élevée de ces produits en fer, 9 a $20 \mathrm{mg}$ de Fe pour $100 \mathrm{~g}$. ce qui semblerait leur conferer une certaine valeur antianémique.

Quant à la teneur en lipides, qui dépend évidemment de l'espèce de poisson utilisé, du mode de traitement industriel ef des divers ingrédients ajoutés, elle ne dépasse pas 5 p. 100 dans les 3 poudres $P A, P B, P C$ et par suite leur rancissement rapide $n$ 'est pas à craindre ; par contre les granulés GD, GE, GF en renferment de 9 à 15 . 100, mais il faut tenir compte de cette forme de conditionnement qui offre une surface de contact avec l'air moins considérable que les poudres. Une telle structure doit pouvoir s'opposer partiellement aux phénomènes d'oxydation ef d'acidification des lipides et limiter dans une certaine mesure les risques d'altération de ces gronulés par rancissement.

Par ailleurs, la forte teneur de ces granulés de poisson en lipides explique leur valeur calorifique élevée : 346 à 368 calories four 100 grammes valeur comparable à celle ce la farine de l'OVIPARU (371 calories 100 g), alors que le pouvoir énergétique des poudres PA, PB, PC fortement délipidées est compris entre 301 et 320 calories pour 100 grammes, soit environ 3 fois $1 / 2$ plus que celui des poissons d'origine.

Le taux d'humidité de ces farines revêt une grande importance, car il conditionne dans une large mesure, les possibilités de stockage. Dès que la teneur en eau augmente, certains microorganismes trouvent dans le substrat protéique de ces farines un terrain favorable à leur développement, il en résulte des altérations des caractères organoleptiques et bactériologiques comme nous le verrons dans un instant. Selon les normes proposées plus haut, une bonne conservation peut être assurée lorsque l'humidité de ces farines ne dépasse pas 10 p. 100, chiffre ramené à 5 p. 100 pour les meilleures qualités.

D'une façon générale, les pourcentages d'humidité des granulés de poisson étudiés (GD, GE, $G F$ ) sont plus faibles que ceux des poudres (PA, PB, PC) :

\begin{tabular}{|c|c|}
\hline Granulés GD & 4,98 p. 100 d'eau \\
\hline Granulés GE & 6,07 p. $100 \mathrm{~d}$ 'eau \\
\hline Poudre PA. & 6,72 p. 100 d'eau \\
\hline Granulés GF & 8,21 p. 100 d'equ \\
\hline Poudre PB. & 9,52 p. 100 d'eau \\
\hline Poudre PC. & 11,10 p. 100 d'eav \\
\hline
\end{tabular}

\section{c) Qualité bactériologique. Conditionnement et stockage}

Parallèlement, alors que dans chaque catégorie de produits examinés - granulés ou poudres - les 2 échantillons les moins humides correspondent à des denrées alimentaires de bonne qualité bactériologique (Tableau III. Poudre PA ef PB-Granulés GD et GE), il est à remarquer que l'échantillon contenant le plus fort pourcentage d'eau est bactériologiquement sovillé :

Granulés GF : présence de Flavobocterium.

et surtout poudre PC: présence d'Aerobacter cerogenes.

Les 6 échantillons étudiés étaient conditionnés dans des sacheis transparents en polyéthylène. Maineureusement, l'obturation de ces emballages réalisée a chaud avec un petit fer à souder n'a pas été obtenue dans des conditions permettant une étanchéité parfaite. Or, non seulement les farines de poisson sont très hygroscopiques mais de plus au Sud-Viet Nam le degré hygrométrique de l'air dépasse souvent 90 . Ces faits suffisent à expliquer la valeur reiativement éle- 
vée des taux d'humiditě observés, bien que dans l'ensemble ils soient inférieurs à la limite prescrite de 10 p. 100.

Enfin, même en supposant que l'ensachage soit effectué de façon correcte, nous pensons que le temps de stockage de ces farines de poisson ne devrait pas excéder un mois, surtout si elles sont conservées dans des locaux non conditionnés. Ces délais nous semblent justifiés, car il ne faut pas oublier qu'aux risques d'altération résultant d'une augmentation du pourcentage d'humidité, viennent s'ajouter, par suite de la perméabilité préférentielle du polyéthylène pour l'oxygène; les possibilités de rancissement ef d'acidification de ces denrées. On peut redouter, semble-t-il dans ces conditions, une oxydation lente des lipides, même si les pourcentages en matières grasses sont inférieurs aux normes prescrites.

\section{d) Essais d'acceptabilité}

Les diverses tentatives poursuivies en Afrique pour introduire les farines de poisson dans les rations de populations carencées en protéines animales, n'ont pas toujours été couronnées de succès. Comme l'ont écrit M. LASSANCE et ses collaborateurs, l'introduction d'un aliment nouveau se heurte aux tendances psychosensorielles qui règlent le comportement alimentaire habituel d'une population. Le heurt psychologique engendré est d'autant plus profond que l'aliment est inconnu ou présenté sous une forme nouvelle qui. ne rappelle en rien son aspect primitif (6). De plus il ne faut pas oublier que certaines peuplades africaines ne consomment que très peu de poisson, voire pas du tout parfois à cause d'interdit religieux. Ceci explique les réticences observée's chez ces groupements humains à l'égard des farines de poisson, en dépit des efforts déployées par les administrations locales pour les présenter sous des formes faciles à mélanger à leur nourriture habituelle.

Au Viet-Nam, bien qu'il soit 'encore trop tôt pour préjuger l'avenir des farines de poisson, i. semble que le Vietnamien, ichtyophage par nature, les acceptera favorablement. Toutefois, il ne faudra pas négliger de faire connaître aux populations intéressées la valeur hautement nutritive de ces denrées. II conviendra aussi d'indiquer aux ménagères; aux responsables des cantines civiles et militaires, aux gestionnaires des hôpitaux et des établissements scolaires les façons les plus adéquates d'introduire ces farines dans les mets de leurs rationnaires (soupes au riz, aux vermicelles, préparations oryzées... additionnées d'un dixième au plus de farine de poisson). Enfin et surtout, les industriels responsables, sans nuire à la qualité des produits fabriqués, devront sans cesse améliorer et rationaliser leurs méthodes de production afin de pouvoir livrer à la consommation des farines de poisson d'un prix aussi modique que possible.

\section{III. - SAUCISSES DE POISSON}

II s'agit là d'une forme de transformation et de conservation des produits de la pêche, tout à fait nouvelle et peut être inédite, car dans la littérature spécialisée que nous avons consultée, il n'est fait nulle mention des saucisses de poisson.

Celles que nous avons examinées, d'un poids voisin de "100 grammes, présentaient une chair rosée tirant sur le brun clair. Elles sont livrées à la consommation sous un emballage en plastique (karalon) épousant exactement la forme de la saucisse.

\section{a) Mode de fabrication}

Ces saucisses de poisson sont fabriquées à partir de la chair de 2 espèces de poisson Notopterus notopterus (fig. no 4) Notopteridae (nom vernacu-

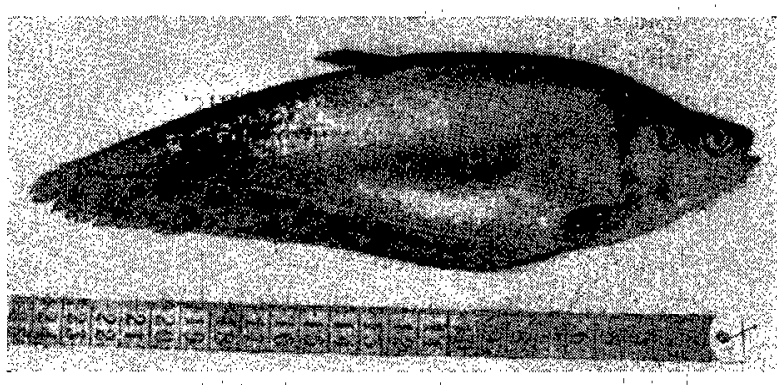

Fig. 4. - Notopterus notopterus (Ca that-lat)

laire vietnamien: (a that-lat) et Chirocentrus dorab (fig. no 5) Chirocentridae (nom vernaculaire vietnamien : Ca rua), d̀ raison d'une partie de Ca that-lat pour 2 parties de Ca rua, la première espèce étant destinée à donner. plus de malléabilité à la pâte. En outre, ce mélange permet d'obtenir, tout en conservant la valeur ali- 
TABIEAU IV

Saucisses de poisson - Caractères bactériologiques et chimiques. Valeur énergétique.

\begin{tabular}{|c|c|c|c|}
\hline Eahantillans & $\begin{array}{c}\text { S } 4 \\
\text { Saucisse de poisson }\end{array}$ & $\begin{array}{c}\text { S B } \\
\text { Saucisse de poisson }\end{array}$ & Poissons vietramiens \\
\hline$\frac{\text { Enmen baotériologique }}{\text { (ospèces microbiennes identifiées) }}$ & Baoillus & Baoillus & $!$ \\
\hline $\begin{array}{l}\text { Pramen ohimique } \\
\text { (Résultats rapportés } \\
\text { à } 100 \text { grammes de produit) } \\
\text { Hurijidité (en g) } \\
\text { Protides (en g) } \\
\text { Lipides (en g) } \\
\text { Glucides (en g) } \\
\text { Cendres minérales (en g) } \\
\text { Chlorures (en g de ClNa) } \\
\text { Phosphore (en mg de P) } \\
\text { Calcium (en mg de Ca) } \\
\text { Rapport Ce/P } \\
\text { Fer (en ms de Fe) }\end{array}$ & $\begin{array}{c}68,04 \\
20,50 \\
1,21 \\
6,96 \\
3,29 \\
1,70 \\
181 \\
38 \\
0,21 \\
c, 066\end{array}$ & $\begin{array}{c}68,26 \\
19,87 \\
2,56 \\
6,12 \\
3,19 \\
1,64 \\
169 \\
30 \\
0,17 \\
0,045\end{array}$ & $\begin{array}{c}\text { Composition ohimique } \\
\text { moyenne } \\
\begin{array}{c}70-85 \\
10-25 \\
0,1-13 \\
\text { moins de } 1 \\
0,8-1,5 \\
0,14-0,36 \\
116=256 \\
7-114 \\
0,03-0,80 \\
0,3-1\end{array}\end{array}$ \\
\hline (en calories et rapportée à 100 grames) & 120,73 & $127, \infty$ & $55-175$ \\
\hline $\begin{array}{l}\text { Polds net de la saucisse } \\
\text { Celorie per saucisse }\end{array}$ & $\begin{array}{c}98 \text { 8 } \\
109,62 \mathrm{cal} \text {. }\end{array}$ & $\begin{array}{c}110 \mathrm{~g} \\
139,70 \mathrm{cal} .\end{array}$ & । \\
\hline
\end{tabular}

mentaire du produit final, une denrée d'un prix : modique : le kilogramme de chair de Ca that lat valent 50 piastres vietnamiennes, alors que le kilo-

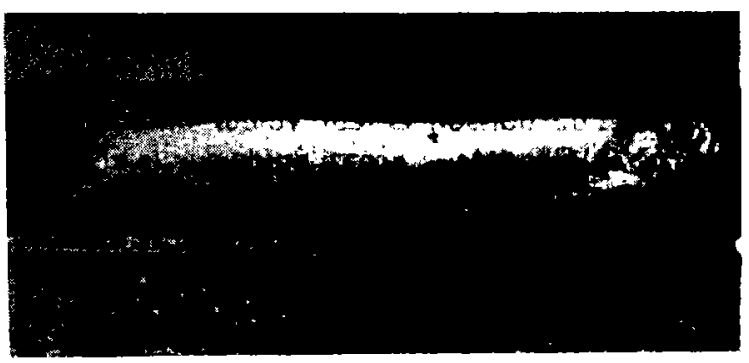

Fig. 5. - Chirocentrus torab (Ca rua).

gramme de Ca rua ne coute que 25 piastres vietnamiennes $(*)$.

(*) En 1960, 100 pinstres viefnamiennes volen environ 6,5 nouveaux francs au marché libre.
Trois machines sont nécessaires à la fabrication industrielle de ces saucisses :

- la Fish scaling machine débarrasse le poisson de ses écailles.

- la Mixing and grinding machine le broye et le malaxe. A la pâte obtenue, on incorpore les produits suivants : sel, poivre en poudre, sucre, piment pulvérisé, oignon hâché, lard de porc, farine d'arrow-root, glutamate monosodique, quelques gouttes de cérosote et un colorant alimentaire.

- enfin la Meat Stuffer permet d'introduire la pâte composée dans de petits sachets en karalon (variété de polyéthylène). Ces sachets possèdent une forme cylindrique, d'un diamètre de 3 à $5 \mathrm{~cm}$., leur longueur est réglable à volonté.

Lorsqu'ils sont pleins de pâte de poisson, on les obture à leurs deux extrémités à l'aide de petifes ficelles, souples et solides.

La stérilisation de ces saucisses est assurée 
par une cuisson dans l'eau chaude à $90^{\circ}$ pendant 60 à 90 minutes. Au bout de ce temps, elles sont plongées dans de l'cau glacée additionnée de quelques gouttes d'eau de Javel. II est à noter que l'étanchéité du Karalon empêche tout risque d'imbibition. Le refroidissement brusque a pour but d'éviter une surcuisson et supprime pratiquement les possibilités d'une réinfection, que l'on pourrait craindre si l'on procédait à un refroidissement lent de ces saucisses.

\section{b) Valeur alimentaire ef nutritive}

La composition chimique et la valeur énergétique des 2 échantillons de saucisses de poisson que nous avons étudiés sont rapportées dans le tableau IV (échantillons SA et SB). On peut remarquer que ces saucisses présentent une composition chimique assez semblable à celle de la chair de poisson, mis à part les pourcentages en glucides et en sels minéraux qui sont un peu plus élevés dans les Saucisses de poisson. C'est pour cette raison que nous avons fait figurer, à titre comparatif, dans le tableau IV la composition chimique moyenne des poissons,

De même, l'apport protidique $(20$ p. 100) et le pouvoir calorifique (120 calories/100 g) de ces saucisses correspondent à ceux des espèces de poisson habituellement consommées au VietNam.

\section{c) Qualité bactériologique}

Grâce au traitement de stérilisation détaillé plus haut, les 2 échantillons examinés peuvent être considérés comme des produits de bonne qualité bactériologique (voir tableau IV).

\section{RÉSUMÉ}

Après avoir étudié dans un précédent article la composition chimique et la valeur nutritive ef énergétique des 19 espèces de poisson de consommation courante au Viet-Nam, ainsi que celles des préparations ichtyologiques vietnamiennes traditionnelles: nuoc mam, mam ca et ca kho, les auteurs ont examiné dans la présente note 3 types de produits industriels de transformation ef de conservation du poisson, fabriqués par le service des industries des pêches du gouvernement vietnamien : conserves appertisées, poudres, granulés et saucisses de poisson. Par leur mode de préparation (adjonction de nuoc mam, de sauce de soja, d'épices et d'aromates de la flore locale), ils conviennent aux habitudes culinaires des Vietnamiens. Ces produits permettent de supplémenter leurs rations souvent déficientes en protéines animales et en apport minéral phosphocalcique. Teneurs moyennes respectives en protéines, calcium, phosphore et calories pour 100 grammes : conserves appertisées : $20,18 \mathrm{~g} ; 444 \mathrm{mg} ; 355 \mathrm{mg} ; 166 \mathrm{cal}$. poudres: $64.41 \mathrm{~g} ; 4.433 \mathrm{mg}: 2.486 \mathrm{mg}: 309 \mathrm{cal}$. granulés: $53,83 \mathrm{~g} ; 3.113 \mathrm{mg} ; 2.173 \mathrm{mg} ; 360 \mathrm{cal}$. - soucisses : $20,18 \mathrm{~g} ; 34 \mathrm{mg} ; 175 \mathrm{mg} ; 124 \mathrm{cal}$.

L'avenir seul dira quel accueil sera réservé au Viet-Nam à ces produits nouveaux apportant sous une forme concentrée et stable les vertus alimentaires et nutritives du poisson.

Service technique des industries des pêches et Institut Pasteur du Viet-Nam.

\section{BIBLIOGRAPHIE}

1. CREACH (P.). - Rôle des farines de poisson dans l'alimentation du bétail. $C$. R. Congrès des pêches et des pêcheries dans I'U. F. d'O. M. Inst. colon. Marseille 1950, p. 298-307.

2. GULLLERM (J.).-Le nuoc mam et l'industrie saumurière en Indochine. Arch. Inst. Pasteur Indochine 1928, no 7. p. 21-61.

3. GUILLOT. - Le poisson dans l'alimentation de l'armée. C. R. Congrès intern: d'étude sur le rôle du poisson dans l'alimentation. Inst. océanogr. Paris, octobre 1950, p. 400-415.

4. JACQUOT (R.) et CREACH (P:): - Les protides du poisson et leur valeur alimentaire. C. R. Congrès intern. d'étude sur le rôle du poisson dans l'alimentation: Inst. océanogr. Paris, octobre 1950 , p. $11-58$.

5. LAFONT (R.). - Formes d'utilisation pour l'alimentation des produits de la pêche dans les eaux continentales du Cambodge. $C$. R. Congrès des pêches et des pêcheries dans I'U. F. d'O. M. Inst. colon. Marseille, 1950, p. $232-236$.

6. LASSANCE (M.), BERVOETS (W:) etEVRARD (G.). - L'avenir des farines de poisson au Congo Belge. Essais d'acceptabilité. Ann. Soc. belge Méd. trop. 1958. 38 : 669-679. 
7. MONNIER (E.). - Les préparations à base de graines de soja. Bull. écon. Indochine, 1935, 38 : 66-85.

8. NGUYEN THI LAU et RICHARD (C.). Le poisson dans l'alimentation du Vietnamien. Rev. Elev. Méd. vét. Pays trop. 1959. । $12(3): 313-24$.

9. ROELS (O.). - La poudre de poisson frais déshydraté pour l'alimentation de
I'homme. Bull. agric. Congo Belge 1957, 48 : 423-438.

10. TOURY (J.). - O. R. A. N. A., Dakar. Communication personnelle.

11. VIALARD GOUDOU (A.), - Etude bactériologique, chimique et valeur alimentaire de la sauce vietnamienne nuoc mam. C. R. $8^{e}$ Congrès des sciences du Pacifique. Manille 1953.

\section{SUMMARY}

Fish as an item of diet in Vietnamien.

(Second Part)

Having stated in the first article, the chemical composition, and nutritional and energy values of 19 species of fish currently consumed in Viet Nam, as well as of certain traditional fish preparations, the authors pass in this second article to the examination of three types of conserved fish products, prepared industrially by the Fish Industry Service of the Government, viz. Fish powder, fish granules and fish sausages. These can be utilised in the normal culinary methods which include the addition of Soya bean sauce, spices and local aromatic herbs, and will supplement the ration, frequently deficient in animal protein and phosphorus-calcium content. Details of composition and calorific values are given. These new products supply in a concentrated and stable form, the particular nutritive items of piscine origin, but only the future can tell whether they will be welcome to the populace.

\section{RESUMEN}

\section{El pescado en la alimentación del pueblo vietnamita (2à parte).}

Después de haber estudiado en un anterior escrito la composicion química y los valores nutritivo y energético de 19 especies de pescado de consumo corriente en Viet-Nam, así como sus preparados ictiológicos tradicionales: nuoc-mam. mam ca y co seho. los autores. continuando su linea de trabajo nos presentan un estudio muy completo e interesante sobre unos productos industriales a base de pescado fabricados por el Servicio de Industrias de la Pesca del Gobierno vietnamita : conservas aperitivos, horinas, granulados y salchichas de pescado.

Su preparación (agregan nuoc mam, salsa de soja, especias y substancias aromáticas de la flöra local) es adecuada a las costumbres culinarias de los vietnamitas. Estos productos permiten suplementar sus raciones, que corrientemente son déficientes en proteinas de origen anima!, calcio y fósforo. Su composición media en proteínas, calcio, fosforo y calorías por 100 gramos de alimento es la siguiente :

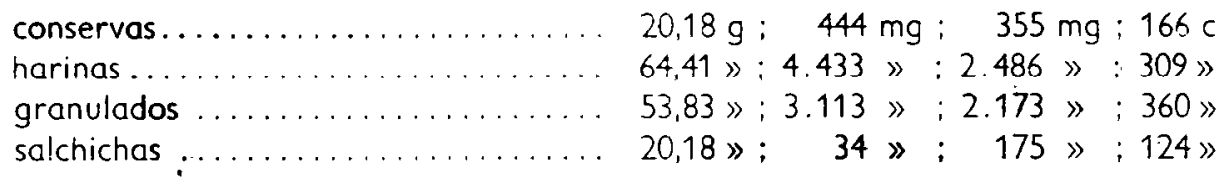

Ya veremos como el pueblo vietnamita acoje estos nuevos productos que proporcionan bajo una forma concentrada y esiable los materiales nutritivos del pescada. 